\title{
Reduction of superfluid gap by scattering
}

\author{
P. Bożek ${ }^{*}$ \\ National Superconducting Cyclotron Laboratory, \\ and Department of Physics and Astronomy, \\ Michigan State University, East Lansing, MI-48824 \\ and \\ Institute of Nuclear Physics, PL-31-342 Kraków, Poland
}

(Dated: November 5, 2018)

\begin{abstract}
The effect nucleon dressing by scattering on the pairing gap in nuclear matter is discussed. Numerical results from self-consistent T-matrix calculations are compared to quasi-particle approximations. The dominant effect of scattering can be accounted for by a renormalization of the quasi-particle strength. The exponential sensitivity of the pairing gap and the critical temperature on the pairing strength makes it strongly dependent on many-body effects.

PACS numbers: $21.65+\mathrm{f}, 24.10 \mathrm{Cn}, 26.60+\mathrm{c}$
\end{abstract}

\section{INTRODUCTION}

Nuclear systems at low temperatures undergo a superfluid phase transition. This is observed in finite nuclei as the even-odd staggering of masses and is expected to occur in infinite nuclear matter inside neutron stars. Calculations in finite nuclei usually assume an effective pairing interaction fitted to the available data. Calculations in nuclear matter are using predominately the bare nucleon-nucleon interaction in the gap equation.

Due to the short range repulsive core in the nuclear potentials Brueckner type ladder resummation of the interaction in medium is necessary. On the other hand, the effective interaction in the gap equation should be two-particle irreducible, i.e. without resummation of the particle-particle (and hole-hole) ladder 11. Thus a good starting point could be the bare nucleon-nucleon interaction, the same as used in the ladder approximation for the two-particle correlation.

The value of the pairing gap depends on nucleon single particle energies. Using single-particle energies obtained from Hartree-Fock or Brueckner-Hartree-Fock (BHF) calculation a different value of the paring gap is obtained. It can be understood as a modification of the effective mass, and consequently a change in the density of states at the Fermi energy. Obviously the value the effective mass is an inherent part of effective parameterizations of the pairing interactions used in calculations in finite systems.

Only relatively few works discuss possible in medium modifications of the pairing interactions beyond a change in the effective mass. Polarization corrections to the bare nucleon-nucleon interactions were discussed in Refs. [2]. It was found that the screening reduces the pairing gap by a factor $\simeq 3$. Another in medium modification was analyzed in the framework of self-consistent nuclear matter calculations [3]. Self-consistent nuclear matter calculations use off-shell nucleon propagators in the resummation of ladder diagrams for the self-energy. In this way a self-consistent spectral function can be obtained. It was found that the use of full spectral functions in the gap equation leads to a strong reduction of the critical temperature and of the superfluid gap in comparison to the quasi-particle approximation. It is the goal of the present paper to identify the main cause of this modification of superfluid properties of nuclear matter and to propose a renormalization of the quasi-particle approximation for the gap equation. In Sect. II we discuss modifications of the two-particle correlations in the normal phase. This enables us to calculate the critical temperature, at which long range two-particle pairing correlations appear. The critical temperature is estimated using full spectral functions and in the renormalized quasi-particle approximation. In Sect. III we present a discussion of the gap equation with full spectral function and compare it to its quasi-particle limit and to the usual mean-field gap equation. In the concluding section $[\mathrm{IV}$ we identify the most important renormalization of the pairing interaction due to off-shell propagation and indicate its consequences for realistic nuclear matter calculations.

*Electronic address: bozek@solaris.ifj.edu.p 


\section{QUASI-PARTICLE LIMIT OF SELF-CONSISTENT LADDER RESUMMATION}

Nuclear medium is a relatively dense system of particles strongly interacting on short distances. Brueckner resummation of particle-particle ladder diagrams defines the so called in medium G-matrix

$$
<\mathbf{p}|G(\mathbf{P}, \Omega)| \mathbf{p}^{\prime}>=V\left(\mathbf{p}, \mathbf{p}^{\prime}\right)+\int \frac{d^{3} q}{(2 \pi)^{3}} V(\mathbf{p}, \mathbf{q}) \frac{\left(1-f\left(\omega_{p_{1}}\right)\right)\left(1-f\left(\omega_{p_{2}}\right)\right)}{\Omega-\omega_{p_{1}}-\omega_{p_{2}}}<\mathbf{q}|G(\mathbf{P}, \Omega)| \mathbf{p}^{\prime}>
$$

where $\mathbf{p}_{\mathbf{1}, \mathbf{2}}=\mathbf{P} / 2 \pm \mathbf{q}$. G-matrix resummation allows to define single particle energies and gives relatively good results for the saturation properties of nuclear matter. In the above equation and in the following we skip the spin, isospin indices which are implicitly summed over. The above equation corresponds to a resummation of particleparticle ladders, with medium effects entering through the Pauli blocking factors $1-f\left(\omega_{p}\right)$ in the numerator and single-particle energies $\omega_{p}$ in the denominator. Most advanced calculations in the Brueckner scheme use the so called continuous choice for the single particle energies $\omega_{p}$, self-consistently defined by the G-matrix 挋.

Another approach starts from the T-matrix approximation for the two-particle correlations [5, 6]. In this scheme the ladder diagrams include both particle particle and hole-hole propagation. The Pauli blocking factor $\left(1-f\left(\omega_{p_{1}}\right)\right)(1-$ $\left.f\left(\omega_{p_{2}}\right)\right)$ in the G-matrix equation is replaced by $1-f\left(\omega_{p_{1}}\right)-f\left(\omega_{p_{2}}\right)$ in the equation for the retarded T-matrix

$$
<\mathbf{p}|T(\mathbf{P}, \Omega)| \mathbf{p}^{\prime}>=V\left(\mathbf{p}, \mathbf{p}^{\prime}\right)+\int \frac{d^{3} q}{(2 \pi)^{3}} V(\mathbf{p}, \mathbf{k}) \frac{\left(1-f\left(\omega_{p_{1}}\right)-f\left(\omega_{p_{2}}\right)\right)}{\Omega-\omega_{p_{1}}-\omega_{p_{2}}+i \epsilon}<\mathbf{q}|T(\mathbf{P}, \Omega)| \mathbf{p}^{\prime}>
$$

The imaginary part of the retarded self-energy in the T-matrix approximation is

$$
\operatorname{Im} \Sigma(p, \omega)=\int \frac{d^{3} k}{(2 \pi)^{3}}<(\mathbf{p}-\mathbf{k}) / 2\left|\operatorname{Im} T\left(|\mathbf{p}+\mathbf{k}|, \omega_{k}+\omega\right)\right|(\mathbf{p}-\mathbf{k}) / 2>_{A}\left(f\left(\omega_{k}\right)+b\left(\omega+\omega_{k}\right)\right),
$$

where $b(\omega)$ is the Bose distribution. $\langle\ldots\rangle_{A}$ denotes antisymmetrization of the T-matrix (also in the spin, isospin indices not explicitly show). The real part of the self energy consists of the Hartree Fock self-energy and a dispersive contribution obtained from $\operatorname{Im} \Sigma$

$$
\operatorname{Re} \Sigma(p, \omega)=\Sigma_{H F}(p)+\mathcal{P} \int \frac{d \omega^{\prime}}{\pi} \frac{\operatorname{Im} \Sigma\left(p, \omega^{\prime}\right)}{\omega^{\prime}-\omega} .
$$

The imaginary part of the self-energy is usually neglected leading to the quasi-particle approximation for the twonucleon propagator in the T-matrix (Eq. 2).

Allowing for off-shell propagation of nucleons and taking the self-energy self-consistently (also its imaginary part) requires the use of full spectral functions in the calculation resulting in a more complicated expressions for the T-matrix and the self-energy [8]

$$
\begin{aligned}
<\mathbf{p}|T(\mathbf{P}, \Omega)| \mathbf{p}^{\prime}>= & V\left(\mathbf{p}, \mathbf{p}^{\prime}\right) \\
& +\int \frac{d \omega_{1}}{2 \pi} \int \frac{d \omega_{2}}{2 \pi} \int \frac{d^{3} q}{(2 \pi)^{3}} V(\mathbf{p}, \mathbf{q}) \frac{\left(1-f\left(\omega_{1}\right)-f\left(\omega_{2}\right)\right)}{\Omega-\omega_{1}-\omega_{2}+i \epsilon} A\left(p_{1}, \omega_{1}\right) A\left(p_{2}, \omega_{2}\right)<\mathbf{q}|T(\mathbf{P}, \Omega)| \mathbf{p}^{\prime}>(5)
\end{aligned}
$$

and

$$
\operatorname{Im} \Sigma^{+}(p, \omega)=\int \frac{d \omega_{1}}{2 \pi} \int \frac{d^{3} k}{(2 \pi)^{3}} A\left(k, \omega_{1}\right)<(\mathbf{p}-\mathbf{k}) / 2\left|\operatorname{Im} T\left(\mathbf{p}+\mathbf{k}, \omega+\omega_{1}\right)\right|(\mathbf{p}-\mathbf{k}) / 2>_{A}\left(f\left(\omega_{1}\right)+g\left(\omega+\omega_{1}\right)\right)
$$

Equations (5), (6) and (4) have to be solved iteratively with the constraint on the assumed density $\rho$ [3, 8]

$$
\rho=\int \frac{d \omega}{2 \pi} \int \frac{d^{3} p}{(2 \pi)^{3}} A(p, \omega) f(\omega)
$$

Results of the self-consistent calculation and of the quasi-particle approximation are very different. In medium cross sections for nucleon-nucleon scattering are smaller when using off-shell nucleons [7]. Also the critical temperature is strongly reduced when using full spectral functions [3].

The T-matrix approximation for the two-particle propagator is directly related to the superfluid gap properties by the Thouless criterion [9] and the condition for long range order [10, 11. On the other BHF calculation using the G-matrix are are much developed for realistic interactions. In this work we derive an improved gap equation which could use the results of advanced G-matrix calculations as an input. 


\section{A. Thouless criterion for superfluidity}

The critical temperature can be obtained from the Thouless criterion for superconductivity [9]. This is the temperature where a singularity in the T-matrix appears at twice the Fermi energy $(\Omega=0)$ and zero total momentum of the pair $(P=0)$. It means that the real part of the inverse T-matrix develops a zero-eigenvalue at the critical temperature

$$
\int \frac{d^{3} p^{\prime}}{(2 \pi)^{3}}<\mathbf{p}\left|\operatorname{Re} T^{-1}(\mathbf{P}=\mathbf{0}, \omega=0)\right| \mathbf{p}^{\prime}>\Delta\left(\mathbf{p}^{\prime}\right)=0 .
$$

It is equivalent to the existence of a nontrivial solution of the gap equation at $T_{c}$

$$
\begin{aligned}
& \Delta(\mathbf{p})= \\
& -\int \frac{d^{3} k}{(2 \pi)^{3}} \int \frac{d \omega}{2 \pi} \int \frac{d \omega^{\prime}}{2 \pi} V(\mathbf{p}, \mathbf{k}) \frac{A\left(k, \omega-\omega^{\prime}\right) A(k, \omega)\left(1-f\left(\omega-\omega^{\prime}\right)-f\left(\omega^{\prime}\right)\right)}{\omega} \Delta(\mathbf{k}) .
\end{aligned}
$$

The two propagators in the gap equation above enter with the full spectral function. On the other hand the BCS quasi-particle gap equation is

$$
\Delta(\mathbf{p})+\int \frac{d^{3} k}{(2 \pi)^{3}} V(\mathbf{p}, \mathbf{k}) \frac{\left(1-2 f\left(\zeta_{k}\right)\right)}{2 \zeta_{k}} \Delta(\mathbf{k})=0
$$

with $\zeta_{p}=p^{2} / 2 m+\Sigma_{H F}(p)-\mu$, and it corresponds to the Thouless criterion for the quasi-particle T-matrix, i.e. the appearance of a singularity in the T-matrix given by Eq. (2). Because of additional averaging over spectral functions in the gap equation with off-shell propagators, a different value of critical temperature comes out.

The self-consistent T-matrix calculation was done for a simple, S-wave, interaction and compared to the quasiparticle approximation for the gap equation [3]. At a density of 0.45 of normal nuclear density $\rho_{0}$ it was found that the critical temperature was reduced from $T_{c}=5 \mathrm{MeV}$ in the mean-field gap equation with Hartree-Fock single particle energies to $T_{c}=1.6 \mathrm{MeV}$ in the T-matrix approximation with off-shell propagation [3]. Below $T_{c}$ a modified T-matrix resummation was used in Ref. [3]. The resulting superfluid gap is significantly smaller than the one obtained from the usual gap equation with quasiparticles. Modifications to the gap equation below $T_{c}$ coming from the use of full spectral functions will be discussed in Sect. [II]. Here we concentrate on the T-matrix equations in the normal phase, which is sufficient for the calculation of the critical temperature. The simple Yamaguchi S wave interaction 3 does not permit calculations at low temperature for normal density nuclear matter.

\section{B. Renormalized quasi-particle interactions}

Excitations in the Fermi liquid close to the Fermi energy can be described by quasi-particles. Quasi-particles are propagating on shell with dispersion relation modified by the presence of the medium. Also the scattering amplitudes between quasi-particles are modified by the medium. One of these modification comes from the quasi-particle limit in the propagator of two nucleons [1, 12. Two particle Green's function can be formally written as a resummation of particle-particle (and hole-hole) ladder diagrams starting from in medium two-particle irreducible vertex [1, 13. For short range interactions the two-particle irreducible vertex can be approximated in the lowest order by the bare nucleon-nucleon interaction, leading to the T-matrix equation (5). It should be pointed out that due to the instantaneous form of the interaction in the T-matrix equation, the full T-matrix depends only on the total energy, and its equation takes a simple form for the retarded T-matrix (Eq. 5), also at finite temperature.

Close to the Fermi energy the spectral function becomes peaked around the quasi-particle pole

$$
A(p, \omega)=Z_{p} 2 \pi \delta\left(\omega-\omega_{p}\right)+R(p, \omega)
$$

where

$$
Z_{p}=\left(1-\left.\frac{\partial \operatorname{Re} \Sigma(p, \omega)}{\partial \omega}\right|_{\omega=\omega_{p}}\right)^{-1}
$$

and $R(p, \omega)$ is the regular part, smooth in the vicinity of the quasi-particle pole. The retarded propagator of two nucleons appearing in the T-matrix ladder can be written as

$$
\frac{\left(1-f\left(\omega_{1}\right)-f\left(\omega_{2}\right)\right)}{\Omega-\omega_{1}-\omega_{2}+i \epsilon} A\left(p_{1}, \omega_{1}\right) A\left(p_{2}, \omega_{2}\right)=B^{r e g}\left(p_{1}, \omega_{1}, p_{2}, \omega_{2}, \Omega\right)
$$




$$
+(2 \pi)^{2} \delta\left(\omega_{1}-\omega_{p_{1}}\right) \delta\left(\omega_{2}-\omega_{p_{2}}\right) \frac{Z_{p_{1}} Z_{p_{2}}\left(1-f\left(\omega_{p_{1}}\right)-f\left(\omega_{p_{2}}\right)\right)}{\Omega-\omega_{p_{1}}-\omega_{p_{2}}} .
$$

$B^{r e g}$ describes the part of the propagator of two nucleons which cannot be written as propagation of two quasi-particles, it originates from the background part $R(p, \omega)$ of the spectral function. Besides this contribution, the propagator of two-nucleons differs from the one used in the quasi-particle T-matrix (2) by the presence of renormalization factors $Z_{p}$ (Eq. 12). The T-matrix with quasi-particle propagators takes the following renormalized form

$$
\begin{aligned}
<\mathbf{p}|T(\mathbf{P}, \Omega)| \mathbf{p}^{\prime}>= & <\mathbf{p}\left|V^{r e n}(\mathbf{P}, \Omega)\right| \mathbf{p}^{\prime}> \\
& +\int \frac{d^{3} q}{(2 \pi)^{3}}<\mathbf{p}\left|V^{r e n}(\mathbf{P}, \Omega)\right| \mathbf{q}>Z_{p_{1}} Z_{p_{2}} \frac{\left(1-f\left(\omega_{p_{1}}\right)-f\left(\omega_{p_{2}}\right)\right)}{\Omega-\omega_{p_{1}}-\omega_{p_{2}}+i \epsilon}<\mathbf{q}|T(\mathbf{P}, \Omega)| \mathbf{p}^{\prime}>,
\end{aligned}
$$

with the renormalized interaction $V^{r e n}$ given by

$$
<\mathbf{p}\left|V^{r e n}(\mathbf{P}, \Omega)\right| \mathbf{p}^{\prime}>=V\left(\mathbf{p}, \mathbf{p}^{\prime}\right)+\int \frac{d \omega_{1}}{2 \pi} \int \frac{d \omega_{2}}{2 \pi} \int \frac{d^{3} q}{(2 \pi)^{3}} V(\mathbf{p}, \mathbf{q}) B\left(p_{1}, \omega_{1}, p_{2}, \omega_{2}, \Omega\right)<\mathbf{q}\left|V^{r e n}(\mathbf{P}, \Omega)\right| \mathbf{p}^{\prime}>
$$

The bare interaction is renormalized by contributions from background parts of the spectral functions, this involves integration over energies far from the quasi-particle pole. The background part of the spectral function is of course necessary to recover sum rules for the particle strength. However, the full treatment of the renormalized interaction is difficult and in the following we replace it by the bare interaction $V^{r e n} \simeq V$ in the gap equation. The only remnant of the dressing of nucleons in medium are the single particle energies

$$
\omega_{p}=\xi_{p}+\operatorname{Re} \Sigma\left(p, \omega_{p}\right)
$$

and the $Z_{p}$ factors in the homogeneous term in the T-matrix equation. It will turn out that these are the dominant modifications responsible for shifting the critical temperature. In the vicinity of the pole the T-matrix equation is dominated by the homogeneous term. In this region the interaction for quasi-particles is effectively renormalized by a factor $Z_{p_{1}} Z_{p_{2}}$.

\section{Two-particle pole with renormalized interactions}

According to the Thouless criterion, a pole in the T-matrix at the Fermi energy $(\Omega=0)$ means that superfluid long range order sets in (Sect. II A). As mentioned earlier, standard mean-field gap equation uses the following kernel

$$
V(p, k) \frac{\left(1-2 f\left(\zeta_{k}\right)\right)}{2 \zeta_{k}}
$$

with mean-field single particle energies $\zeta_{k}=k^{2} / 2 m+\Sigma_{H F}(k)-\mu$. It leads to a critical temperature $T_{c}=5 \mathrm{MeV}$. One could take single particle energies $\omega_{p}$ beyond Hartree-Fock, e.g. from BHF or T-matrix calculations. However, the difference between the resulting effective masses is of the order $Z_{p}$. Hence we stay at the order of Hartree-Fock effective mass in the standard gap equation, including the modified single particle energies (16) only together with $Z_{p}$ factor renormalization.

The second estimate for the critical temperature is obtained from the condition of appearance of the pole in the T-matrix with full self-consistent spectral functions. It is equivalent to the following kernel in the gap equation

$$
\int \frac{d \omega}{2 \pi} \int \frac{d \omega^{\prime}}{2 \pi} V(\mathbf{p}, \mathbf{k}) \frac{A\left(k, \omega-\omega^{\prime}\right) A(k, \omega)\left(1-f\left(\omega-\omega^{\prime}\right)-f\left(\omega^{\prime}\right)\right)}{\omega}
$$

where $A(p, \omega)$ is obtained from full self-consistent calculation of normal nuclear matter at finite temperature with off-shell propagators in the T-matrix ladder [8]. It gives a very different value for $T_{c}=1.6 \mathrm{MeV}$ at $\rho=.45 \rho_{0}$.

Finally we can use the renormalized interaction strength in the homogeneous term of the T-matrix equation (Eq. 14). It is equivalent to using a renormalized interaction in the kernel of the quasi-particle gap equation

$$
V(p, k) Z_{p}^{2} \frac{\left(1-2 f\left(\omega_{k}\right)\right)}{2 \omega_{k}},
$$

with the single particle energies $\omega_{p}$ and $Z_{p}$ factors obtained from the full self-consistent T-matrix calculation (Eq. 16). It gives a value of $T_{c}=2.2 \mathrm{MeV}$, much closer to the result of the calculation with full spectral functions. The $Z$ factor obtained from the full self-energy is not close to 1 , at the Fermi energy we find in our model calculation [3] $Z_{p_{f}} \simeq 0.7$. Such a small value of the renormalization factor can explain the large difference in critical temperatures found in the standard quasi-particle gap equation and in the one with full spectral function. 


\section{GAP EQUATION WITH DRESSED PROPAGATORS}

\section{A. Gap equation with full spectral function}

Below the critical temperature a nonzero solution of the gap equation is possible. The kernel of the gap equation is very similar to the two-particle propagator in the T-matrix resummation (Eq. 9). However one of the propagators includes the normal self-energy as well as the off-diagonal one $\Delta(p)$ [3]. The full retarded propagator can be expressed using the normal propagator $G(p, \omega)$ (which includes only the normal self-energy)

$$
G_{s}(p, \omega)=\frac{1}{G(p, \omega)^{-1}+|\Delta|^{2}(p) G^{\star}(p,-\omega)} .
$$

As a result in the kernel of the gap equation

$$
\Delta(\mathbf{p})=-\int \frac{d^{3} k}{(2 \pi)^{3}} \int \frac{d \omega}{2 \pi} \int \frac{d \omega^{\prime}}{2 \pi} V(\mathbf{p}, \mathbf{k}) \frac{A\left(k, \omega-\omega^{\prime}\right) A_{s}(k, \omega)\left(1-f\left(\omega-\omega^{\prime}\right)-f\left(\omega^{\prime}\right)\right)}{\omega} \Delta(\mathbf{k}) .
$$

two spectral functions $A(p, \omega)=-2 \operatorname{Im} G(p, \omega)$ and $A_{s}(p, \omega)=-2 \operatorname{Im} G_{s}(p, \omega)$ appear. Both spectral functions can be calculated knowing the two self-energies $\Sigma(p, \omega)$ and $\Delta(p)$. The off-diagonal self-energy, ie. the superfluid gap can be obtained from the gap equation (21). On the other hand the normal self-energy cannot be calculated in the usual T-matrix approximation as above $T_{c}$. This is related to the appearance of the Cooper instability in the two-particle propagator in the ladder approximation.

The T-matrix equation can be modified by introducing also anomalous propagators in the ladder 11]. In Ref. [3] we used a simpler modification of the ladder diagrams, using a mixed ladder with one full propagator and one with only normal self-energy included. This means that the singularity of the T-matrix which appears at $T_{c}$ at the Fermi energy $\Omega=0$ and zero total momentum of the pair, stays there also below $T_{c}$. This reflects the presence of long range order 10, 11]. In such a way we were able to obtain self-consistent solutions for the normal self-energy in the ladder approximation and for the superfluid gap in the mean-field approximation, using at all stages full spectral functions $A(p, \omega)$ and $A_{s}(p, \omega)$, without quasi-particle approximation. The results for the superfluid gap at several temperatures below $T_{c}$ are indicated by triangles in Fig. 1. This calculation cannot be extended straightforwardly to zero temperature because at the Fermi energy the imaginary part of the self-energy vanishes and the discretization of the spectral functions is not possible close to the Fermi energy. At the Fermi momentum quasi-particles appear with small width.

By comparing the imaginary part of the self-energy at two temperatures $T=1.7 \mathrm{MeV}$ and $1.2 \mathrm{MeV}$ one notices that the only modification is the reduction of single-particle width close to the Fermi energy with temperature, in agreement with general properties of Fermi liquids. Thus we can take as an approximation a temperature independent imaginary part of the self-energy. We proceed by taking the imaginary part of the self-energy $\operatorname{Im} \Sigma(p, \omega)$ as calculated at $T=1.63 \mathrm{MeV}$, slightly above $T_{c}$. Obviously the dispersive contribution to the real part of the self-energy is also fixed (4). The Hartree-Fock energy is obtained from

$$
\Sigma_{H F}(p)=\int \frac{d^{3} k}{(2 \pi)^{3}} V(|\mathbf{p}-\mathbf{k}| / 2,|\mathbf{p}-\mathbf{k}| / 2) n(p)
$$

where the momentum distribution is given by

$$
n(p)=\int \frac{d \omega}{2 \pi} A_{s}(p, \omega) f(\omega) .
$$

The shift of the Fermi energy from the value $\omega=0$ at $T=1.63 \mathrm{MeV}$ to keep the density

$$
\rho=.45 \rho_{0}=\int \frac{d^{3} p}{(2 \pi)^{3}} n(p)
$$

constant when decreasing the temperature to zero is only $0.2 \mathrm{MeV}$ and has negligible effect on the Hartree-Fock energy. Thus the main change in the self-energy when decreasing the temperature occurs in the off-diagonal part $\Delta(p)$. At any given temperature the superfluid gap is obtained from the gap equation with full spectral function (21). Where the dependence of the kernel of the gap equation on $\Delta(p)$ enters through the spectral function

$$
A_{s}(p, \omega)=-2\left(\left(\omega+\xi_{p}+\operatorname{Re} \Sigma^{+}(p,-\omega)\right)^{2} \operatorname{Im} \Sigma^{+}(p, \omega)+\operatorname{Im} \Sigma^{+}(p,-\omega) \Delta^{2}(p)+\left(\operatorname{Im} \Sigma^{+}(p,-\omega)\right)^{2} \operatorname{Im} \Sigma(p, \omega)\right) /
$$




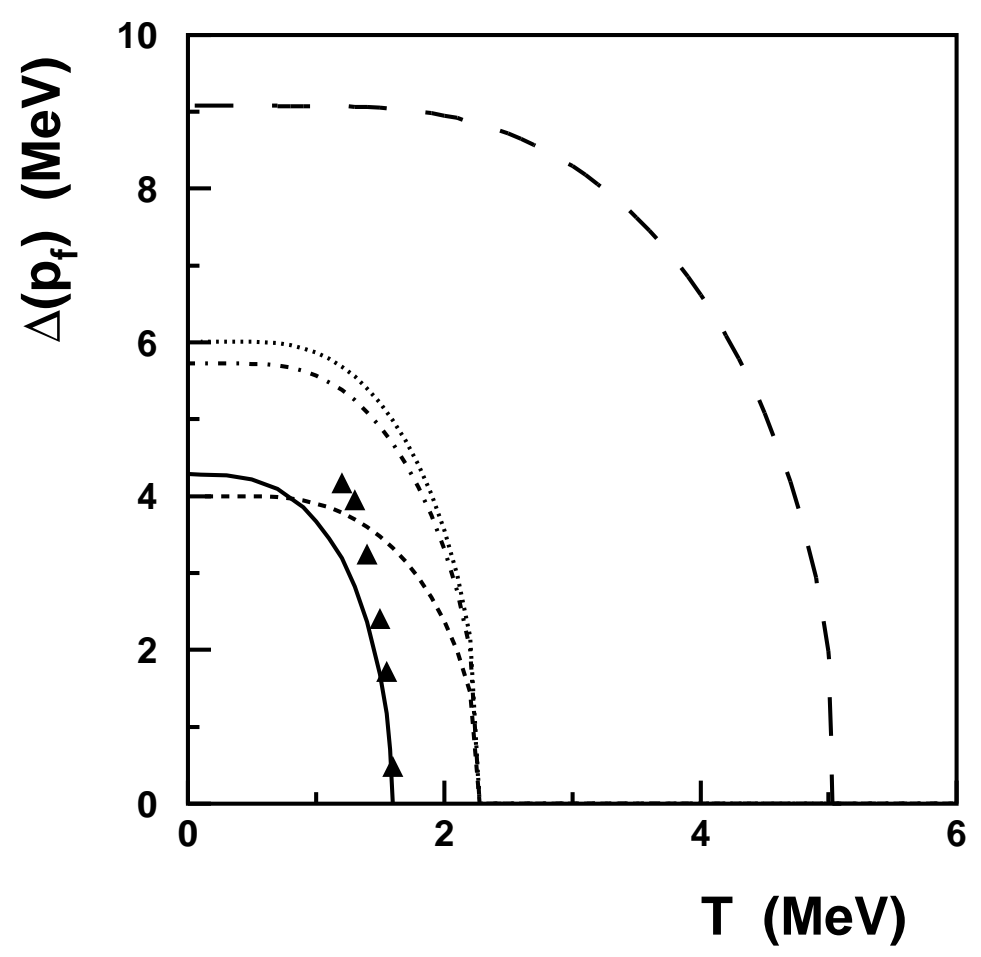

FIG. 1: Superfluid gap at the Fermi momentum as function of the temperature at $\rho=.45 \rho_{0}$. Points denote results obtained from self-consistent solution of the ladder self-energy and gap equations. The solid line represents results obtained using the imaginary part of the self-energy fixed at $T=1.63 \mathrm{MeV}$ and solving self-consistently the Hartree-Fock and the full gap equation (21). The long dashed line is the mean-field quasi-particle solution of the gap equation with Hartree-Fock single-particle energies (26). The dashed line is the result of the quasi-particle approximation (36) without renormalizing the superfluid gap (38). The dashed-dotted and dotted lines are the results of the quasi-particle approximation (36) with renormalization of the superfluid gap by $Z_{p}(42)$ and by $1 /\left(1-O\left(p, \omega_{p}\right)\right)$ (41) respectively.

$$
\begin{aligned}
& \left(\left(\left(\omega-\xi_{p}-\operatorname{Re} \Sigma^{+}(p, \omega)\right)\left(\omega+\xi_{p}+\operatorname{Re} \Sigma^{+}(p,-\omega)\right)-\operatorname{Im} \Sigma^{+}(p, \omega) \operatorname{Im} \Sigma^{+}(p,-\omega)-\Delta^{2}(p)\right)^{2}\right. \\
& \left.+\left(\operatorname{Im} \Sigma^{+}(p, \omega)\left(\omega+\xi_{p}+\operatorname{Re} \Sigma^{+}(p,-\omega)\right)+\operatorname{Im} \Sigma^{+}(p,-\omega)\left(\omega-\xi_{p}-\operatorname{Re} \Sigma^{+}(p, \omega)\right)\right)^{2}\right)
\end{aligned}
$$

In the above equation $\operatorname{Im} \Sigma$ is fixed and temperature independent, according to our approximation, and $R e \Sigma$ depends only very weakly on the temperature and the superfluid gap through the Hartree-Fock energy. The solution of the gap equation using a fixed single particle width down to zero temperature is represented by the solid line in Fig. 11. As expected close to the temperature where the imaginary part of the self-energy was fixed $(T=1.63 \mathrm{MeV})$ the result is close to the fully self-consistent solution denoted by the triangles on the figure. As the temperature is lowered some deviations appear. It is due to the decrease of the single particle width close to the Fermi energy in the fully self-consistent solution (Fig. 2). In the following we will compare the solution of the gap equation with full spectral function to the corresponding quasi-particle approximation. Since the imaginary part of the self-energy is fixed at $T=1.63 \mathrm{MeV}$, the properties of the quasi-particle pole $\left(\omega_{p}\right.$ and $\left.Z_{p}\right)$ are taken from the self-consistent solution at the same temperature. Our aim is to explain the big difference between the mean-field solution with on-shell propagators (long-dashed line in Fig. 11) and the gap equation with full spectral function below $T_{c}$ (solid line). For this purpose it is sufficient to compare the approximation with nontrivial but fixed spectral properties with its quasi-particle limit.

The mean-field BCS gap equation is

$$
\Delta(\mathbf{p})+\int \frac{d^{3} k}{(2 \pi)^{3}} V(\mathbf{p}, \mathbf{k}) \frac{\left(1-2 f\left(E_{k}\right)\right)}{2 E_{k}} \Delta(\mathbf{k})=0,
$$




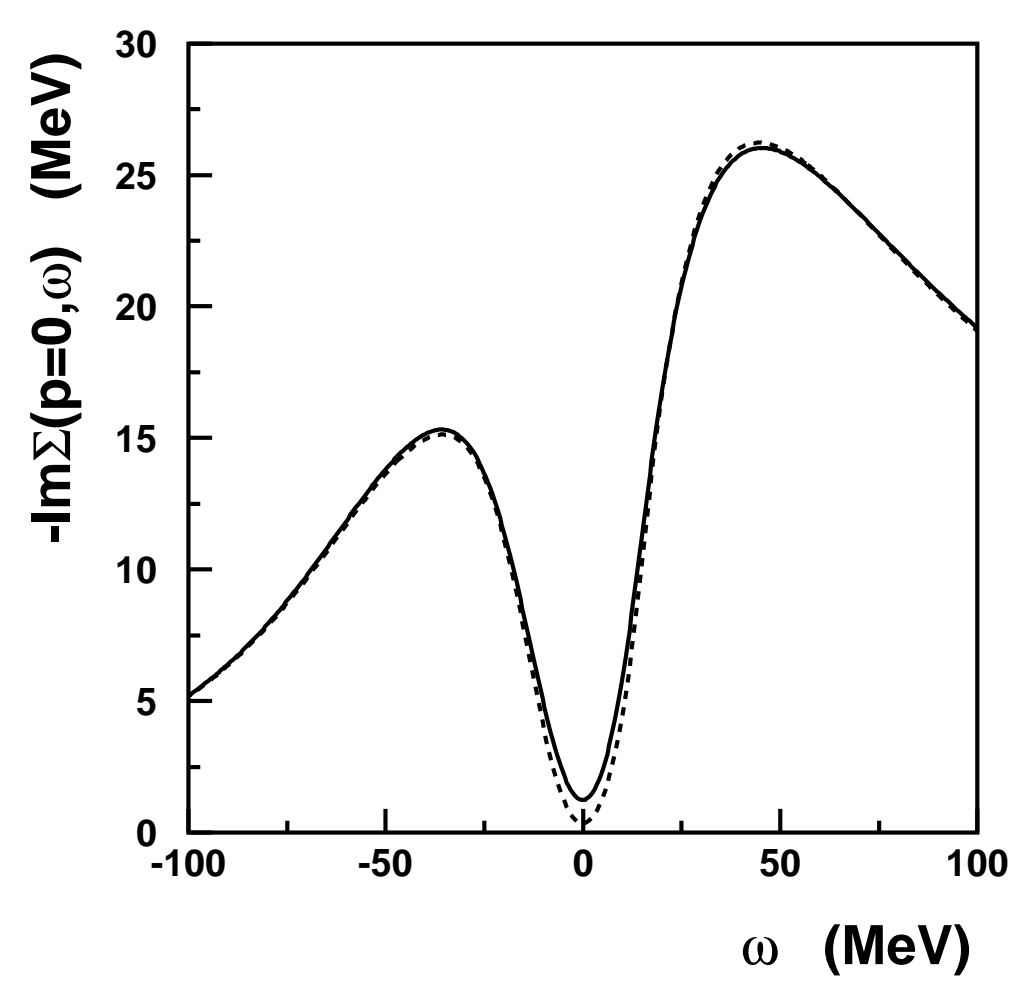

FIG. 2: Imaginary part of the self-energy at zero momentum for two temperatures $T=1.7$ (solid line)and $1.2 \mathrm{MeV}$ (dashed line).

where $E_{k}=\sqrt{\zeta_{k}^{2}+\Delta(k)^{2}}$. It gives significantly larger values for the superfluid gap (long-dashed line in Fig. 1) than the full solution (solid line) in all ranges of temperatures below $T_{c}$.

\section{B. Quasi-particle approximation for the superfluid spectral function}

Above $T_{c}$ we have used the standard form of the quasi-particle approximation for the spectral function $A(p, \omega)=$ $2 \pi Z_{p} \delta\left(\omega-\omega_{p}\right)$. Using it we have found a significant reduction of the critical temperature with respect to the mean-field approximation. To obtain a quasi-particle approximation for the kernel of the gap equation (21) with full spectral function we have to construct an approximation for the superfluid spectral function $A_{s}$. We can write the spectral function (25) putting an infinitesimally small imaginary part of the self-energy $(\operatorname{Im} \Sigma(p, \omega)=-i \eta)$

$$
\begin{aligned}
& A_{s}(p, \omega)=-2 \operatorname{Im}\left(\left(\omega+\xi_{p}+\operatorname{Re} \Sigma(p,-\omega)+i \eta\right) /\left(\left(\omega-\xi_{p}-\operatorname{Re} \Sigma(p, \omega)\right)\right.\right. \\
& \left.\left.\left(\omega+\xi_{p}+\operatorname{Re} \Sigma(p,-\omega)\right)+\Delta^{2}(p)+i \eta(2 \omega-\operatorname{Re} \Sigma(p, \omega)+\operatorname{Re} \Sigma(p,-\omega))\right)\right)
\end{aligned}
$$

with $\xi_{p}=p^{2} / 2 m-\mu$. It is useful to define the even $S(p, \omega)=\operatorname{Re} \Sigma(p, \omega)+\operatorname{Re} \Sigma(p,-\omega)$ and odd $\omega O(p, \omega)=$ $\operatorname{Re} \Sigma(p, \omega)-\operatorname{Re} \Sigma(p,-\omega)$ parts of the real part of the self-energy with respect to the Fermi energy [14]. Notice that we can take $S(p, 0)=0$ redefining $\mu$. We have

$$
A_{s}(p, \omega)=-2 \operatorname{Im}\left(\frac{\omega(1-O(p, \omega))+\xi_{p}+S(p, \omega)}{((1-O(p, \omega))-E(p, \omega))(\omega(1-O(p, \omega))+E(p, \omega))+i \eta \omega(1-O(p, \omega))}\right),
$$

where

$$
E(p, \omega)=\sqrt{\left(\xi_{p}+S(p, \omega)\right)^{2}+\Delta^{2}(p)}
$$


The above expression has poles at

$$
\pm \omega=\epsilon_{p}=\frac{E\left(p, \epsilon_{p}\right)}{1-O\left(p, \epsilon_{p}\right)}=\frac{E_{p}}{1-O_{p}},
$$

which gives two quasi-particle contributions to the spectral function on both sides of the Fermi energy

$$
A_{s}(p, \omega)=2 \pi Z_{p}^{\prime}\left(\frac{E_{p}+\xi_{p}+S_{p}}{2 E_{p}} \delta\left(\omega-\epsilon_{p}\right)+\frac{E_{p}-\xi_{p}-S_{p}}{2 E_{p}} \delta\left(\omega-\epsilon_{p}\right)\right),
$$

with $S_{p}=S\left(p, \epsilon_{p}\right)$ and

$$
Z_{p}^{\prime}-1=\left(1-\left.\frac{\partial(E(p, \omega) /(1-O(p, \omega)))}{\partial \omega}\right|_{\omega=\epsilon_{p}}\right)\left(1-O_{p}\right) .
$$

It will be useful to relate this new pole renormalization strength to the usual renormalization factor $Z_{p}$ (Eq. 12). We can write

$$
Z_{p}^{\prime}{ }^{-1}=\left(1-\left.\frac{\partial \Sigma(p, \omega)}{\partial \omega}\right|_{\omega=\operatorname{sign}\left(p-p_{f}\right) \epsilon_{p}}\right)+\left.\frac{E_{p}-\operatorname{sign}\left(p-p_{f}\right)\left(\xi_{p}+S_{p}\right)}{E_{p}} \frac{\partial S(p, \omega)}{\partial \omega}\right|_{\omega=\epsilon_{p}},
$$

with $\operatorname{sign}(x)=\Theta(x)-\Theta(-x)$. For small values of the superfluid gap the superfluid quasiparticle position $\operatorname{sign}\left(p-p_{f}\right) \epsilon_{p}$ is very close to the position of the pole in the normal propagator $\omega_{p}=\xi_{p}+\operatorname{Re} \Sigma\left(p, \omega_{p}\right)$. Substituting $\omega_{p}$ for the energy argument in the first term in Eq. (31) we get

$$
Z_{p}^{\prime}{ }^{-1}-Z_{p}^{-1}=\left.\frac{E_{p}-\operatorname{sign}\left(p-p_{f}\right)\left(\xi_{p}+S_{p}\right)}{E_{p}} \frac{\partial S(p, \omega)}{\partial \omega}\right|_{\omega=\epsilon_{p}}
$$

The first factor on the right hand side of the above equation is small except close to the Fermi energy where the second factor precisely vanishes. Thus we expect that the new quasi-particle pole renormalization factor $Z_{p}^{\prime}$ can be approximated by the renormalization factor of the normal spectral function $Z_{p}$ corresponding to the same momentum. A numerical calculation confirms this to a very good accuracy (Fig. [).

The spectral function takes the form

$$
A_{s}(p, \omega)=2 \pi Z_{p}\left(u_{p}^{2} \delta\left(\omega-\epsilon_{p}\right)+v_{p}^{2} \delta\left(\omega-\epsilon_{p}\right)\right),
$$

with coherence factors

$$
u_{p}^{2}\left(v_{p}^{2}\right)=\frac{\epsilon_{p}+(-)\left(\xi_{p}+S_{p}\right) /\left(1-O_{p}\right)}{2 \epsilon_{p}} .
$$

These coherence factors can be very well approximated by an expression similar to the one used in the usual mean-field gap equation

$$
u_{p}^{2}\left(v_{p}^{2}\right)=\frac{\epsilon_{p}+(-) \omega_{p}}{2 \epsilon_{p}}
$$

The approximation works to within $1 \%$ for $\Delta\left(p_{f}\right)<10 \mathrm{MeV}$.

Substituting the quasi-particle expressions for the spectral functions $A$ and $A_{s}$ into the kernel of the gap equation one obtains

$$
\Delta(p)=-\int \frac{d^{3} k}{(2 \pi)^{3}} Z_{k}^{2} V(p, k) \frac{\left(1-2 f\left(\epsilon_{p}\right)\right)}{2 \epsilon_{p}} .
$$

We have obtained an expression for the gap equation with a kernel very similar to the mean-field BCS one (Eq. 17), but with the interaction renormalized by $Z_{p}^{2}$ and a different quasi-particle energy $\epsilon_{p}$.

In the limit of vanishing gap $\Delta(p) \longrightarrow 0$ all quasi-particle approximations for the gap equation reduce to the Thouless condition for the renormalized T-matrix equation (Eq. 19). Accordingly we have the same condition for $T_{c}$ as discussed in Sect. II . 


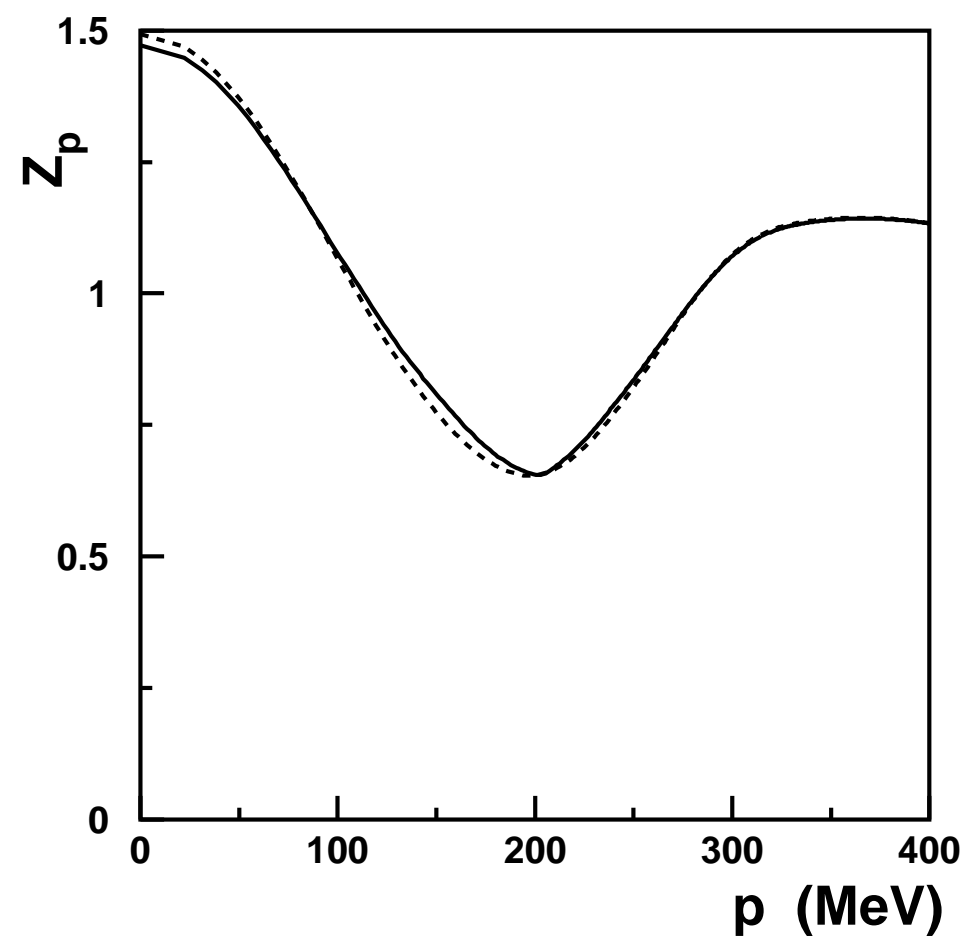

FIG. 3: Quasi-particle pole renormalization factor for the full spectral function $Z_{p}^{\prime}$ (Eq. 30) as function of momentum (solid line) compared to the quasi-particle renormalization in the normal spectral function $Z_{p}$ (Eq. 12) (dashed line) for $\Delta\left(p_{F}\right)=2 \mathrm{MeV}$.

\section{Quasi-particle energies}

In order to relate our quasi-particle gap equation (36) to usual approaches we have to obtain an approximation for the quasi-particle energies

$$
\epsilon_{p}=\frac{\sqrt{\left(\xi_{p}+S_{p}\right)^{2}+\Delta^{2}(p)}}{1-O_{p}}
$$

To extract the positions $\pm \epsilon_{p}$ of the poles of the spectral function one has to know the real part of the self energy for energies on both sides of the Fermi energy. We would like to obtain an expression which could be used as a correction to calculations using quasi-particle approximation like BHF.

In BHF approaches one calculates a single particle energy which includes dispersive corrections to the position of the quasi-particle pole (Eq. 16) in the usual spectral function $A(p, \omega)$. A simple approximation would be to use $\omega_{p}$ instead of $\zeta_{p}$ in the expression for the quasi-particle energies in the superfluid

$$
\epsilon_{p}=\sqrt{\omega_{p}^{2}+\Delta^{2}(p)}
$$

Clearly the above expression does not give the right value for the energy gap at the Fermi momentum (Fig. 国). The energy gap at $p_{F}$ should be

$$
\epsilon_{p_{F}}=\frac{\Delta\left(p_{F}\right)}{1-O_{p_{F}}}=Z_{p_{F}} \Delta\left(p_{F}\right)
$$

Let us define a renormalized energy gap

$$
\tilde{\Delta}(p)=\frac{\Delta(p)}{1-O_{p}}
$$




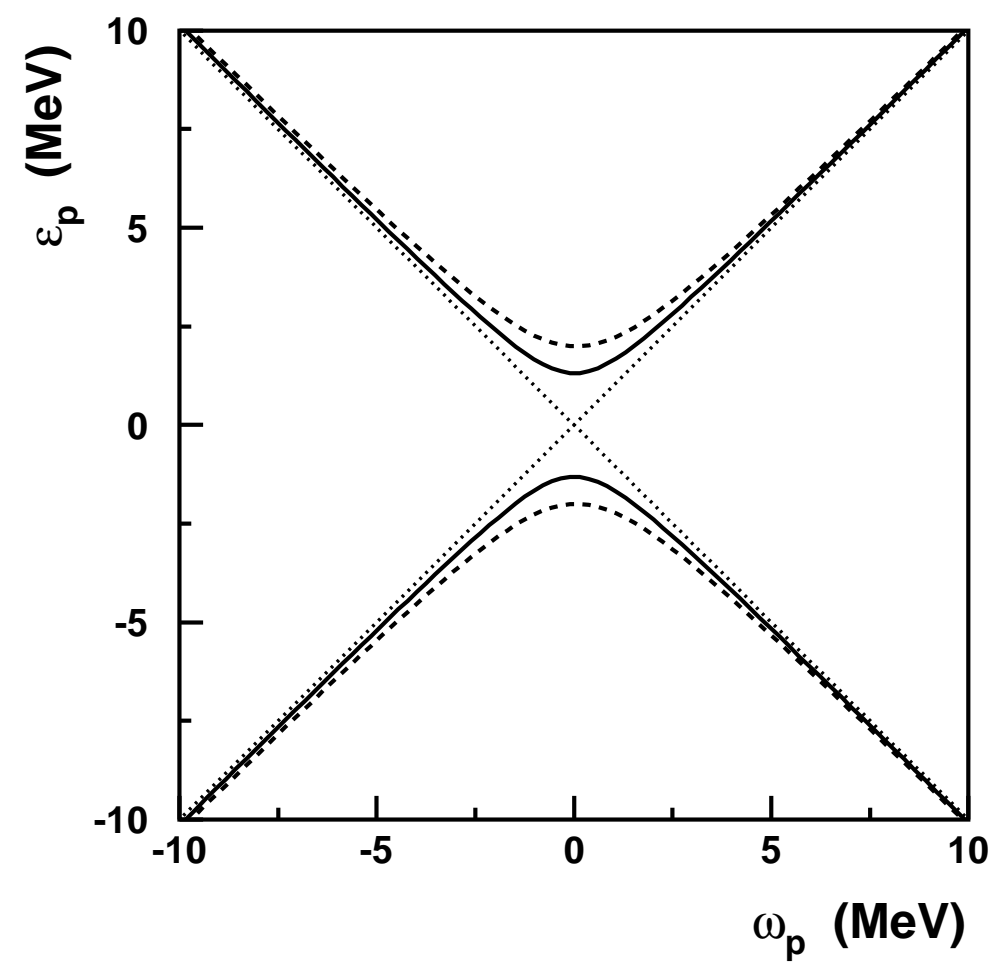

FIG. 4: Position of the superfluid quasi-particle pole $\epsilon_{p}$ (solid line) as function of quasi-particle energy compared to the naive expression $\sqrt{\omega_{p}^{2}+\Delta^{2}(p)}$ (dashed line) (Eq. 38) for $\Delta\left(p_{f}\right)=3 \mathrm{MeV}$. The quasi-particle energies obtained using renormalized superfluid gap $\sqrt{\omega_{p}^{2}+\Delta(p)^{2} /\left(1-O_{p}\right)^{2}}$ (Eq. 40) cannot be distinguished from the solid line on the scale of the figure.

Results for the quasi-particle poles using this renormalization of the superfluid order parameter

$$
\epsilon_{p}=\sqrt{\omega_{p}^{2}+\tilde{\Delta}^{2}(p)}
$$

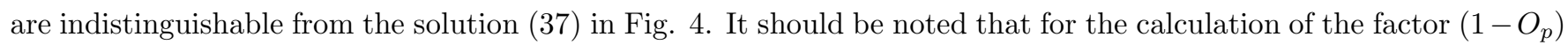
we can take $O_{p}=O\left(p, \epsilon_{p}\right) \simeq O\left(p, \omega_{p}\right)$. So that the approximation for $\epsilon_{p}$ can be expressed using the quasi-particle pole energy $\omega_{p}$ and the values of the real part of the self energy at $\pm \omega_{p}$. However, usually in BHF calculations one does not calculate the factor $1-O_{p}$, since the value and the derivative of the real part of the self-energy is known only at $\omega_{p}$ and not at $-\omega_{p}$. In this case the position of the poles in the superfluid can be approximated by

$$
\epsilon_{p}=\sqrt{\omega_{p}^{2}+\hat{\Delta}^{2}(p)}
$$

with the renormalization of the superfluid gap taken as

$$
\hat{\Delta}(p)=\Delta(p) Z_{p}
$$

$\hat{\Delta}(p)$ is very close to $\tilde{\Delta}(p)$ around the Fermi energy. Some differences appear only around $p=0$ (for the assumed interaction), but this region is less important for the solution of the gap equation. Again the energies obtained using expression (42) are indistinguishable from the solid line in Fig. 6 .

\section{Results for superfluid gap in quasi-particle approximation}

In previous sections we derived a quasi-particle approximation for the kernel of the superfluid gap equation (Eq. 21). The results of the full gap equation with integration of over the energies in the fermion propagators (solid line 
TABLE I: Values for the critical temperature and superfluid gap at zero temperature for different approximations.

\begin{tabular}{l|ccc}
\hline approximation & $T_{c}(\mathrm{MeV})$ & $\Delta\left(p_{F}\right)_{T=0}(\mathrm{MeV})$ & $\Delta\left(p_{F}\right) / T_{c}$ \\
\hline \hline $\begin{array}{l}\text { off-shell gap } \\
\text { equation (21) }\end{array}$ & 1.6 & 4.3 & 2.7 \\
\hline $\begin{array}{l}\text { quasi-particle app. with } \\
\tilde{\Delta}(p) \text { (Eqs. 36, 41) }\end{array}$ & 2.2 & 6.0 & 2.7 \\
\hline $\begin{array}{l}\text { quasi-particle app. with } \\
\hat{\Delta}(p) \text { (Eqs. 36, 42) }\end{array}$ & 2.2 & 5.7 & 2.6 \\
\hline $\begin{array}{l}\text { quasi-particle app. with } \\
\Delta(p) \text { (Eqs. 36, 38) }\end{array}$ & 2.2 & 4.0 & 1.8 \\
\hline $\begin{array}{l}\text { mean-field BCS } \\
\text { (Eq. 26) }\end{array}$ & 5.0 & 10.8 & 2.2 \\
\hline $\begin{array}{l}\text { weak coupling BCS } \\
\text { weak coupling BCS with } \\
\text { renormalized energy gap }\end{array}$ & - & - & 2.5 \\
\hline
\end{tabular}

in Fig. 11) can be compared to quasi-particle results. The dotted line in Fig. 1 represents the solution of the gap equation (36) with renormalization of the energy gap $\tilde{\Delta}(p)$ for the calculation of the superfluid quasi-particle energies (Eq. 41). The dashed-dotted line represents an analogous calculation but with the energy gap $\hat{\Delta}(p)$ (Eq. 43). Both quasi-particle approximation are very close to each other with critical temperature $T_{c}=2.2 \mathrm{MeV}$. The value of $T_{c}$ is the same as obtained in Sect. II C using renormalized interactions in the ladder approximation. This value of critical temperature is much closer to the one obtained from the self-consistent solution $T_{c}=1.6 \mathrm{MeV}$ than was the mean-field value $T_{c}=5 \mathrm{MeV}$. Also the value of the superfluid gap is much closer to the one calculated using full of-shell spectral functions (Table I). The superfluid gap at zero temperature in the quasi-particle approximation $\Delta\left(p_{F}\right)=6 \mathrm{MeV}$ is much closer to the solution of the off-shell gap equation $\Delta\left(p_{F}\right)=4.3 \mathrm{MeV}$ than the one obtained from the mean-field BCS solution $\Delta\left(p_{F}\right)=10.8 \mathrm{MeV}$. From the results in Table If we can notice that the ratio between the superfluid gap at zero temperature and the critical temperature is larger in the solution of the full gap equation (21) and in quasi-particle approximations (36) with renormalization of the energy gap (41 or 42), than in the mean-field solution (26) and in quasi-particle approximations without renormalization of the superfluid energy gap (36, 38). In the weak coupling limit we have 14,15

$$
\frac{\left.\Delta\left(p_{F}\right)\right|_{T=0}}{T_{c}}=\pi e^{-\gamma} \simeq 1.76
$$

If the energy gap $\tilde{\Delta}\left(p_{F}\right)$ is renormalized with respect to the superfluid gap $\Delta\left(p_{F}\right)$ (the off diagonal self-energy) we have

$$
\frac{\left.\Delta\left(p_{F}\right)\right|_{T=0}}{T_{c}}=\frac{\left.\tilde{\Delta}\left(p_{F}\right)\right|_{T=0}\left(1-O_{p_{F}}\right)}{T_{c}}=\frac{\left.\tilde{\Delta}\left(p_{F}\right)\right|_{T=0}}{T_{c} Z_{p_{F}}} \simeq \frac{1.76}{Z_{p_{F}}} \simeq 2.5 .
$$

Ratios of the superfluid gap and of the critical temperature for the solution of the full gap equation and quasi-particle approximations with renormalized superfluid gap is close to $1.76 / Z_{p_{F}}$ (Table $\mathbb{1}$ ). On the other hand mean-field gap equation and quasi-particle approximation without renormalization of $\Delta(p)$ give $\left.\Delta\left(p_{F}\right)\right|_{T=0} / T_{c}$ closer to 1.76 . These relation can be fulfilled only approximately since we are far from the region of applicability of the weak coupling BCS solution.

\section{CONCLUSIONS}

Numerical solution of the gap equation with full spectral functions (21) shows a strong reduction of the superfluid gap and of the critical temperature with respect to the mean-field BCS solution (26). To understand this effect we constructed a quasi-particle approximation for the full gap equation. The effects of nontrivial spectral functions can be approximated using a renormalized strength of the interaction $V(p, k) Z_{k}^{2}$. Also the energy gap in the calculation of the quasi-particle poles in the superfluid is renormalized. For the renormalization of the gap we used two expression 
$\Delta(p) /\left(1-O_{p}\right)$ and $\Delta(p) Z_{p}$. In both cases we obtained results for $\Delta(p)$ and $T_{c}$ much closer to the full solution than the mean-field approximation. Having at one's disposal only the single particle energies $\omega_{p}$ and the $Z_{p}$ factors obtained from a realistic BHF type calculation, the gap equation can be corrected. A reduced interaction strength $V(p, k) Z_{k}^{2}$ must be taken and a factor $Z_{p}$ appears between the energy gap $\hat{\Delta}(p)$ and the off-diagonal self-energy $\Delta(p)$. This is equivalent to solving a gap equation for $\hat{\Delta}(p)$ with reduced interaction $V(p, k) Z_{p} Z_{k}$. In the weak coupling limit $\left.\hat{\Delta}(p)\right|_{T=0} \simeq 1.76 T_{c}$ but $\left.\Delta(p)\right|_{T=0} \simeq 1.76 T_{c} / Z_{p_{F}}$.

In the illustrative model here presented the scattering corrections are very strong $\left(Z_{p_{F}} \simeq .7\right)$ and there are still some differences between the improved quasi-particle approximation and the full solution of the gap equation. We expect that at normal nuclear density where the $Z$ factor is closer to 1, quasi-particle approximation with renormalized interaction strength would be much closer to the full solution. This is the case for neutron matter where effects of renormalization of quasi-particle poles and shifts in single-particle energies are smaller [16]. In future work we plan to investigate the effects of the renormalization of the interaction by background corrections (15).

In this investigation we used the bare potential for the two-particle irreducible vertex. However, it is known that polarization effects reduce the superfluid gap by a factor $\simeq 3[2$. We must conclude that although there are no ladder corrections to the interaction in the gap equation, other many-body effects modify the effective interaction. Due to the exponential dependence of the gap solution on the strength of the interaction, these usually neglected corrections modify strongly superfluid parameters in nuclear matter.

\section{Acknowledgments}

This work was partly supported by the National Science Foundation under Grant PHY-9605207.

[1] A.B. Migdal, Theory of Finite Fermi Systems (John Wiley and Sons, New York, 1967).

[2] J.W. Clark, C.G. Källman, C.H. Yang and D.A. Chakkalakal, Phys. Lett. B61 331 (1976); T.L. Ainsworth, J. Wambach and D. Pines, Phys. Lett. B222 173 (1989); J. Wambach, T.L. Ainsworth and D. Pines, Nucl. Phys. A555 128 (1993); H.-J. Schulze, J. Cugnon, A. Lejeune, M. Baldo and U. Lombardo, Phys. Lett. B375 1 (1996).

[3] P. Bożek, Nucl. Phys. A657 187 (1999).

[4] M. Baldo, I. Bombaci, L.S. Ferreira, G. Giansiracusa and U. Lombardo, Phys. Rev. C 432605 (1990).

[5] B.E. Vonderfecht, W.H. Dickhoff, A. Polls and A. Ramos, Nucl. Phys. A555 1 (1993).

[6] T. Alm, G. Röpke, A. Schnell, N.H. Kwong and S. Köhler, Phys. Rev. C53 2181 (1996); A. Schnell, T. Alm and G. Röpke, Phys. Lett. B387 443 (1996).

[7] W.H. Dickhoff, Phys. Rev. C58 2807 (1998).

[8] P. Bożek, Phys. Rev. C59 2619 (1999).

[9] D.J. Thouless, Ann. Phys. (N.Y.) 10553 (1960).

[10] L.P. Kadanoff and P.C. Martin, Phys. Rev. 124670 (1961).

[11] R. Hausmann, Zeit. Für Phys. B91 291 (1993); R. Hausmann, Phys. Rev. B49 12975 (1994).

[12] P. Nozière, Theory of Interacting Fermi Systems, (New York, Benjamin, 1994)

[13] A.A. Abrikosov, L.P. Gorkov and I.E. Dzyaloshinski, Methods of Quantum Field Theory in Statistical Physics (PrenticeHall, Englewood Cliffs, 1963).

[14] J.R. Schrieffer, Theory of Superconductivity (W.A. Benjamin, Inc., Massachusetts, 1964); G.D. Mahan, Many-Particle Physics (Plenum Press, New York, 1981).

[15] A.L. Fetter, J.D. Walecka, Quantum Theory of Many-Particle Systems, (McGraw-Hill, New York, 1971)

[16] M. Baldo and A. Grasso, nucl-th/003039. 